\title{
Intracytoplasmic Sperm Injection Outcome in Patient at Risk of Poor Responder Using Mild Ovarian Stimulation Protocol versus Short Gonadotropin Releasing Hormone Agonist Protocol \\ Mohamed S. Hasanien, Shoukry A. Elawdy, Amr M. Elerasy \\ Department of Obstetrics and Gynecology, Faculty of Medicine - Al-Azhar University \\ Corresponding author: Amr M. Elerasy; Mobile:01000141386; Email: amroerasy@yahoo.com
}

\begin{abstract}
Background: Poor ovarian response to stimulation in IVF cycles is a challenging and frustrating condition for both clinician and patient, due to its poor prognosis in terms of pregnancies and live births. Although in the literature a large number of papers have been published in which many stimulation protocols suggested should be considered as the best for these women, however, no conclusive results have been reached. Aim of the Work: To evaluate ICSI outcome in patient at risk of poor responder using mild ovarian stimulation protocol versus short gonadotropins releasing hormone agonist protocol. Patients and Methods: This randomized controlled study was conducted on one hundred patients, complaining of infertility at risk of poor responders, patients with at least two of the criteria specific for poor responders at assisted reproductive technology unit, international Islamic center for population studies and research, Alazhar University, Cairo, Egypt, between august 2016 and august 2017. Results: Statistically significant differences were found in the Endometrial thickness, E2 at day of HCG, No. of dominant follicles at day of HCG, Number of retrieved oocyte, fertilization rate, Embryo number, Number of transferred embryos, Pregnancy rate and cost p-value 0.029, 0.036, 0.007, 0.027, 0.048, $0.019,0.046,0.044,0.001$ respectively. Conclusion: ICSI outcome was evaluated in patients at risk of poor responders using short Gonadotropin releasing hormone agonist versus mild ovarian stimulation protocol (clomiphene citrate); showing that short Gonadotropin releasing hormone agonist should be preferred in these patients.
\end{abstract}

Keywords: Sperm Injection Outcome, Poor Responder, Mild Ovarian Stimulation, Short Gonadotropin Releasing Hormone.

\section{INTRODUCTION}

In the field of assisted reproductive technologies great steps forward have been made in recent years in terms of clinical knowledge and technological development especially in IVF laboratories. One of the fundamental steps to reach the success is still related to the number of eggs obtained after hormonal stimulation by gonadotropins in combination with $\mathrm{GnRH}$ analogues ${ }^{(1)}$.

The Bologna ESHREcriteria represent the first real attempt by the scientific community to unify the many definitions proposed to identify poor responder patients by establishing a definite point from which to begin and how to find therapeutic strategies. It was concluded that "poor ovarian responders " should be considered patients having at least two of the following criteria: (1)previous episode of poor ovarian response $(<3$ oocyte)with astandard dose of medication, (2)an abnormal ovarian reserve with AFC $<5-7$ follicles or AMH $<0.5-1.1 \mathrm{ng} / \mathrm{ml}$, (3)women above 40 years of age or presenting other risk factors for poor response such as aprevious ovarian surgery,genetic defects,chemotherapy, radiotherapy and autoimmune disorders ${ }^{(\mathbf{1})}$.

The incidence of poor ovarian responders among infertile women has been estimated at 9-24 $\%$ but according to recent reviews, it seems to be slightly increased. The limitation in quantifying the incidence of these patients among the infertile population is due to the difficulty of a clear definition in literature ${ }^{(2)}$.

Predicting ovarian response before starting hormonal stimulation is the only way to administer an efficient and safe treatment.The most important predictors of the ovarian response to hormonal stimulation are age, biochemical parameters (basal FSH levels in the early follicular phase,serum antimullerian hormone (AMH), and morphological characteristics (antral follicular count $\{\mathrm{AFC}\}$ and ovarian volume) $)^{(2)}$.

Although ovarian reserve declines with age it doesn't represent an optimal predictor of ovarian response ${ }^{(3)}$.

Although many protocols with different doses and types of gonadotropins have been proposed in the literatures over the past 20 years for the management of poor responder patient, yet, there is no really efficient treatment that could solve the problem of poor ovarian response and the current question is still about the ideal protocol for patients defined as 'poor responders'?

\section{Aim of the Study}

To evaluate ICSI outcome in patient at risk of poor responder using mild ovarian stimulation protocol versus short gonadotropins releasing hormone agonist protocol. 


\section{PATIENTS AND METHODS}

This randomized controlled study was conducted at assisted reproductive technology unit, international Islamic center for population studies and research, Alazhar University, Cairo, Egypt, between august 2016 and august 2017. The study was approved by the Ethics Board of Al-Azhar University.

One hundred patients, complaining of infertility at risk of poor responders, Patients with at least two of the following criteria were defined as poor responders:

1-age more than 40 years old;2-basal $\mathrm{FSH}$ (folliclular stimulation hormone) is more than $12 \mathrm{miu} / \mathrm{ml} ; 3$-three or fewer oocyte retrieved in the previous IVF cycle;4-low estradiol level on the day ofHCG(human chorionic gonadotropin) administration $<1500 \mathrm{pmol} / \mathrm{ml}$.

Patients above age of 43 years old, with BMI (body mass index) higher than 30, ultrasound evidence of PCO (polycystic ovary syndrome), endometriosis, myoma, autoimmune disease or metabolic disorder were excluded from the study.

The study protocol was approved by ethical committee. Computer assisted randomization was used. All patients received adequate counseling and signed an informed consent form.

\section{Inclusion criteria}

Age: $<43$ years old

FSH: $<15 \mathrm{miu} / \mathrm{ml}$

BMI: $<30$

No evidence of pco by U/S

No pelvic myoma

No pelvic endometriosis

No autoimmune disease or metabolic disorder

\section{Exclusion criteria}

\section{Clinical assessment:}

Severe semen abnormalities

Full and through careful History taking for proper assessment of the target population for choosing women meeting selection criteria, history included personal, menstrual, obstetric, past history, family history, and history of the present illness.

Complete general examination to exclude presence of any other systemic disease, abdominal examination, and doing comprehensive local pelvic examination during which careful inspection of the external genitalia. Speculum examination of the entire vagina then bimanual assessment of the uterine size and position, also assessment of the presence or absence of adnexal masses.

\section{Laboratory Investigation:}

Semen analysis, basal levels of FSH (follicle stimulating hormone), LH (luetinizing hormone), E2 (estradiol), PRL (prolactin) on day 3 of menstrual cycle, auto-antibodies profile like lupus anticoagulant, anti- cardiolipin antibodies were checked and serumTSH. Preoperative routine investigations was requested $(\mathrm{CBC}$, urine analysis, renal and liver functions as well as $\mathrm{HCV}$ and $\mathrm{HBV}$ screening).

Patients randomly divided into two equal groups:

> Group A: short Gn.R.Hormone Agonist Protocol (50 patients),

$>$ Group B: Mild Ovarian Stimulation protocol (50 patients).

\section{Short Gn.R.Hormone Agonist Protocol (50} patients)

Start Gn.R.hormone Agonist treptoreline 0.1 SC injection (decapeptyl 0.1;ferring pharmaceutical; Germany) from $1^{\text {st }}$ day of menstrual cycle until day of HCG injection, HMG (vial) from $2^{\text {nd }}$ day of cycle. Doses were adjusted according to ovarian reserve.

Daily folliculometry startedfrom the $6^{\text {th }}$ day of the cycleby transvaginal ultrasound scan and blood test to evaluate plasmatic estradiol levels until criteria of HCG injection(at least 3 follicles attain diameter of $18-20 \mathrm{~mm}$ ).

After that, Gonadotropine doses were adjusted according to ovarian response.final oocyte maturation was triggered with $10,000 \mathrm{IU}$ of HCG (choriomon 10, 000, IBSA, Switzerland) when the dominant follicles reached a maximum diameter of 18-20 mm.oocyte retrieval was performed under transvaginal ultrasound control 34-36hr after HCG injection.Intracytoplasmic sperm injection (ICSI) was performed in all cases for all metaphase II oocytes in order to obtain a good fecundation rate and to maximize the chances of embryotransferee.Only empryosgrade $\mathrm{A}$ and $\mathrm{B}$ were transferred. After the transfer all patients received luteal support with progesterone (prontogest $100 \mathrm{mg}$ amp.,IM, IBSA, Switzerland).

$\beta H C G$ assay was carried out in serum after 2 weeks, if positive ultrasound done after 2 weeks to demonstrate clinical pregnancy.

Mild Ovarian Stimulation protocol (50 patients)

Clomiphene citrate $50 \mathrm{mg}$ tablet (clomid $50 \mathrm{mg}$ tab., sanofi, pharmaceutical, multinational) twice daily from $2^{\text {nd }}$ day of menstraual cycle for 5 days, 225 IU HMG (Merional 75 IU vial, IBSA, swizerland)from day 5 ofthe cycle, Cetrorelix 0.25 mg (Cetrotide, Al Mottahedoonpharma) was administered daily when one or more follicles reached $14 \mathrm{~mm}$ in diameter.

Daily folliculometry startedfrom the $7^{\text {th }}$ day of the cycleuntill criteria of HCG injection, then further steps as before in group A. 


\section{Statistical Analysis}

Data were analyzed using Statistical Program for Social Science (SPSS) version 20.0. Quantitative data were expressed as mean \pm standard deviation (SD).

Qualitative data were expressed as frequency and percentage.

\section{The following tests were done:}

- Independent-samples t-test of significance was used when comparing between two means.
- Chi-square $\left(\mathrm{X}^{2}\right)$ test of significance was used in order to compare proportions between two qualitative parameters.

- The confidence interval was set to $95 \%$ and the margin of error accepted was set to 5\%. So, the p-value was considered significant as the following:

- Probability (P-value)

- P-value $<0.05$ was considered significant.

- P-value <0.001 was considered as highly significant.

- P-value >0.05 was considered insignificant.

\section{RESULTS}

Table (1): Comparison between groups according to demographic data and Basel hormones.

\begin{tabular}{|c|c|c|c|c|}
\hline Baseline & $\begin{array}{c}\text { Group A: Short } \\
(\mathbf{N}=\mathbf{5 0})\end{array}$ & $\begin{array}{c}\text { Group B: Mild } \\
\text { Stimulation }(\mathrm{N}=50)\end{array}$ & t-test & p-value \\
\hline \multicolumn{5}{|l|}{ Demographic Data } \\
\hline $\begin{array}{l}\text { Age (years) } \\
\text { Mean } \pm S D \\
\text { Range } \\
\end{array}$ & $\begin{array}{c}34.88 \pm 4.26 \\
27-42\end{array}$ & $\begin{array}{c}35.72 \pm 4.19 \\
25-43\end{array}$ & 1.747 & 0.320 \\
\hline $\begin{array}{c}\text { BMI }\left[\mathbf{w t} /(\mathbf{h t})^{2}\right] \\
\quad \text { Mean } \pm S D \\
\text { Range }\end{array}$ & $\begin{array}{c}24.72 \pm 3.02 \\
18-30\end{array}$ & $\begin{array}{c}25.02 \pm 2.28 \\
20-29\end{array}$ & 1.948 & 0.164 \\
\hline \multicolumn{5}{|l|}{ Basel hormones } \\
\hline $\begin{array}{l}\text { FSH } \\
\quad \begin{array}{l}\text { Mean } \pm S D \\
\text { Range }\end{array} \\
\end{array}$ & $\begin{array}{c}9.29 \pm 2.25 \\
4.7-14.2\end{array}$ & $\begin{array}{c}10.22 \pm 2.58 \\
4.7-14\end{array}$ & 3.707 & 0.057 \\
\hline $\begin{array}{l}\text { LH } \\
\begin{array}{l}\text { Mean } \pm S D \\
\text { Range }\end{array} \\
\end{array}$ & $\begin{array}{c}3.72 \pm 1.25 \\
1.2-7.9 \\
\end{array}$ & $\begin{array}{c}4.49 \pm 1.88 \\
1.2-9.7 \\
\end{array}$ & 5.705 & 0.019 \\
\hline $\begin{array}{l}\text { E2 } \\
\begin{array}{l}\text { Mean } \pm S D \\
\text { Range }\end{array} \\
\end{array}$ & $\begin{array}{c}55.12 \pm 18.44 \\
22-119\end{array}$ & $\begin{array}{c}53.08 \pm 14.29 \\
0.1-164\end{array}$ & 0.219 & 0.241 \\
\hline $\begin{array}{l}\mathbf{A M H} \\
\quad \begin{array}{l}\text { Mean } \pm S D \\
\text { Range }\end{array} \\
\end{array}$ & $\begin{array}{c}0.47 \pm 0.17 \\
0.1-1.2\end{array}$ & $\begin{array}{c}0.45 \pm 0.19 \\
0.01-1.01\end{array}$ & 0.017 & 0.897 \\
\hline $\begin{array}{l}\text { PRL } \\
\begin{array}{l}\text { Mean } \pm S D \\
\text { Range }\end{array} \\
\end{array}$ & $\begin{array}{c}15.05 \pm 7.36 \\
0.5-37.8 \\
\end{array}$ & $\begin{array}{c}13.22 \pm 4.71 \\
1.5-25 \\
\end{array}$ & 2.190 & 0.142 \\
\hline $\begin{array}{l}\text { TSH } \\
\begin{array}{l}\text { Mean } \pm S D \\
\text { Range }\end{array}\end{array}$ & $\begin{array}{c}2.41 \pm 0.52 \\
0.07-11\end{array}$ & $\begin{array}{c}2.50 \pm 0.83 \\
0.5-4.2 \\
\end{array}$ & 0.123 & 0.727 \\
\hline
\end{tabular}

This table shows no statistically significant difference between groups according to demographic data. This table shows statistically significant difference between groups according to LH. 
Table (2): Comparison between groups according to outcome

\begin{tabular}{|c|c|c|c|c|}
\hline & $\begin{array}{c}\text { Group A: Short } \\
(\mathbf{N}=\mathbf{5 0})\end{array}$ & $\begin{array}{c}\text { Group B: Mild } \\
\text { Stimulation }(N=50)\end{array}$ & t-test & p-value \\
\hline End. Thick. At day of HCG & $10.18 \pm 1.83$ & $8.23 \pm 1.70$ & 2.020 & 0.029 \\
\hline E2 at day of HCG & $1449.36 \pm 189.74$ & $1127.96 \pm 146.89$ & 3.354 & 0.036 \\
\hline No. of dominant follicles & $4.21 \pm 1.04$ & $2.94 \pm 0.69$ & 4.691 & $0.007 *$ \\
\hline No. of OPO & $2.52 \pm 1.16$ & $1.83 \pm 0.89$ & 5.205 & 0.027 \\
\hline MI & $31(62 \%)$ & $23(46 \%)$ & 1.973\# & 0.160 \\
\hline MII & $21(42 \%)$ & $14(28 \%)$ & $1.582 \#$ & 0.208 \\
\hline Fertilization & $3.34 \pm 0.45$ & $3.13 \pm 0.50$ & 2.025 & 0.048 \\
\hline No. of Embryo & $1.80 \pm 0.70$ & $1.38 \pm 0.50$ & 2.431 & 0.019 \\
\hline Grade (A) & $27(54 \%)$ & $21(42 \%)$ & $1.022 \#$ & 0.317 \\
\hline Grade $(B)$ & $19(38 \%)$ & $11(22 \%)$ & $2.333 \#$ & 0.127 \\
\hline No. of ET & $1.85 \pm 0.72$ & $1.68 \pm 0.50$ & 2.350 & 0.046 \\
\hline Grade (A) & $25(50 \%)$ & $18(36 \%)$ & 1.469\# & 0.226 \\
\hline Grade (B) & $16(32 \%)$ & $10(20 \%)$ & $1.299 \#$ & 0.254 \\
\hline \multicolumn{5}{|l|}{$\beta H C G$} \\
\hline Positive & $18(36 \%)$ & $11(22 \%)$ & $5.426 \#$ & $0.044 *$ \\
\hline Negative & $7(14 \%)$ & $7(14 \%)$ & & \\
\hline \multicolumn{5}{|l|}{ Clinical Pregnancy } \\
\hline Empty $G S$ & $3(6 \%)$ & $3(6 \%)$ & \multirow{3}{*}{0.475} & \multirow{3}{*}{0.789} \\
\hline Single Viable & $11(22 \%)$ & $6(12 \%)$ & & \\
\hline Twins & $4(8 \%)$ & $2(4 \%)$ & & \\
\hline Cost & $4347.75 \pm 221.74$ & $3836.25 \pm 195.65$ & 12.235 & $<0.001 * *$ \\
\hline
\end{tabular}

This table shows statistically significant difference between groups according to End. Thick.At day of HCG, E2 at day of HCG, No. of dominant follicles, No. of OPO, Fertilization, No. of Embryo, No. of ET, $\beta$ HCG, positive and Cost.

\section{DISCUSSION}

Poor ovarian response to stimulation in IVF cycles is a challenging and frustrating condition for both clinician and patient, due to its poor prognosis in terms of pregnancies and live births. Although in the literature a large number of papers have been published in which many stimulation protocols as the best in these women yet, no conclusive results on this issue have been reached ${ }^{(\mathbf{1})}$.

In this prospective randomized controlled study, we evaluated the ICSI outcome in patients at risk of poor responders using short Gonadotropin releasing hormone agonist versus mild ovarian stimulation protocol (clomiphene citrate). It was concluded that short Gonadotropin releasing hormone agonist should be preferred in these patients.

Our data showed that in poor responder patients a short GnRH agonist protocol gave better results in terms of endometrial thickness, estradiol level, mature oocytes collected, fertilization rate, embryo numbers, number of transferred embryos and clinical pregnancy rate with a comparable amount of gonadotropins used among the two groups.

Our findings revealed in the clomiphene citrate patients that the implantation rate and the clinical pregnancy rate were statistically significantly lower than ones observed in patients treated with short $\mathrm{GnRh}$ agonist protocol.

Recently, for a more patient-friendly IVF with fewer injections and a lower dose of gonadotropins, a new interest in the use of $\mathrm{CC}$, especially in poor responder patients, has been raised. All studies concluded that the mild protocol can be considered a valid alternative for these patients, regarding reduced doses of gonadotropins used and a shorter duration of the stimulation regimen and, therefore, more cost effective and patient friendly than conventional IVF, even though the overall pregnancy and live birth rates remained low in these cases ${ }^{(1,4)}$.

A Cochrane review issued by Gibreelet $\boldsymbol{a l} .^{(5)}$, evaluating the efficacy of $\mathrm{CC}$ with gonadotropins (with or without a mid-cycle antagonist) versus gonadotropins alone in $\mathrm{GnRH}$ agonist protocols in normal responder patients, concluded that there was no evidence to indicate 
that CC differed significantly from the standard protocol in terms of live birth or pregnancy rates.

Other work has evaluated the efficacy of the association of $\mathrm{CC}$ to a high dose of Gonadotropin in 48 patients. In this study improvement in estradiol levels, number of dominant follicles, oocyte retrievel, number of embryos transferred and biochemical pregnancy rates, however, the overall clinical and live birth rate remained low ${ }^{(6)}$.

Schimberniet al. ${ }^{(7)}$ has evaluated the short GnRH agonist versus flexible antagonist versus clomiphene citrate regimen in poor responder undergoing IVF randomized controlled trial 250 poor responders in previous IVF cycle at least 3 months before divided them into three groups: group A: 68 women treated with clomiphene citrate and FSH plus antagonist, Group B: 71 patients treated with FSH plus GnRH antagonist, Group C: 75 patients treated with FSH plus GnRH agonist.

The GnRH agonist protocol showed a significantly higher pregnancy rate $(29.3 \%$ vs. $5.9 \%$ vs. $14.1 \%$ respectively) than the clomiphene and the GnRH antagonist protocol, number of mature oocytes collected, estradiol levels and endometrial thickness. The cost of medications for each baby born was lower for the GnRH agonist protocol than for the others; the implantation rate was significantly lower in the clomiphene group (4.8\%) compared to the $\mathrm{GnRH}$ antagonist group $(9.3 \%)$ and the GnRH agonist groups (19.2\%). No significant differences emerged for total FSH administered, days of stimulation, numbers of oocytes re-trieved and embryos transferred ${ }^{(8)}$.

The GnRH agonist protocol may result in stable and low LH and progesterone (P) levels throughout the stimulation phase and may also cause suppression of endogenous FSH levels, leading to a follicular cohort of all small follicles at the initiation of FSH stimulation resulting in asynchronized follicular development. The advantages of this protocol are increased number of oocytes collected, additional pregnancy chancesfrom cryopreserved embryos, and improvement in patient scheduling ${ }^{(9)}$.

Natural cycle IVF in poor responders has been proposed as an alternative to standard stimulation protocols. This approach appears to be less invasive and less expensive for poor responders who do not show an increase in oocyte production, with standard ovarian stimulation. However, there is a study suggesting that such a strategy is not beneficial for clinical pregnancy rates ${ }^{(\mathbf{1 0})}$.

In contrast, Clomiphene citrate (CC) administration in the early follicular phase with $r$ FSH may improve the outcome of stimulation in poor responders ${ }^{(11)}$.
There are several advantages for $\mathrm{CC}$ used in $\mathrm{COH}$ during IVF:

(1) Reduce Gn administration and lower the cost, (2) have fewer side effects, and (3) prevent ineffective follicles stimulation thereby saving follicle storage ${ }^{(\mathbf{1 2})}$.

There are also some negative judgments for $\mathrm{CC}$ used in $\mathrm{COH}$ both for patients and clinicians. According to some studies, pregnancy outcome were not desirable for patients undergoing $\mathrm{COH}$ protocol with $\mathrm{CC}$, which decreased the number of retrieved oocyte and increased cancellation rate. Its long half-life period and cumulative peripheral antiestrogenic effect could inhibit endometrial growth and decrease the quality and quantity of cervical mucus, resulting as a side-effect on embryo implantation ${ }^{(13)}$.

According to results of study carried out on animals, high concentration of CC could induce oocyte and embryo degeneration ${ }^{(\mathbf{1 4})}$.

Ozcan-Cenksoyet $\boldsymbol{a l}^{\left({ }^{(15)}\right.}$ suggested that for the stimulation of poor responder patients the short protocol is better compared to $\mathrm{GnRH}$ antagonist/aromatase inhibitor letrozole and $\mathrm{GnRH}$ antagonist/ clomiphene citrate protocols regarding maximum estradiol levels, numbers of mature oocytes retrieved and cancellation rate.

During the first two or three days of the menstrual cycle when there are selection and growth of cohort of follicles moving from the primordial to preantralstage,the flare up effect of the short protocol may boost follicle growth increasing the number of follicles growing in the recruited cohort.

\section{CONCLUSION AND RECOMMENDATIONS}

In this prospective randomized controlled study,we evaluated the ICSI outcome in patients at risk of poor responders using short Gonadotropin releasing hormone agonist versus mild ovarian stimulation protocol (clomiphene citrate). It was concluded that short Gonadotropin releasing hormone agonist are preferred for dealing with these patients.

\section{REFERENCES}

1) Ferraretti AP, IaMarca A, Fauser BC, Tarlatzis B, Nargund $G$ andGianaroli L (2011): ESHRE consensus on the definition of "poor response" to ovarian stimulation for in vitro fertilization: Bologna criteria. Hum Reprod.,26:1616-24.

2) Broekmans FJ, Kwee J, Hendriks DJ, Mol BW andLambalk CB (2006): A systematic review of tests predicting ovarian reserve and IVF outcome. Hum Reprod Update, 12: 685 -718.

3) Al-Azemi M, Killick S, Duffy $\mathrm{S}$ et al. (2011):Multi-marker assessment of ovarian 
reserve predicts oocyte yield after ovulation induction,Human Reproduction, 26(2):414-422.

4) Kyrou D, koLiBianakisem, VenetisCa, PaPanikoLaoueg, Bontis $\mathbf{J}$ andtarLatzis BC (2009): How to improve the probability of pregnancy in poor responders undergoing in vitro fertilization: a systematic review and a metaanalysis. FertilSteril., 91: 749-766.

5) GibreelA, MaheshwariA and Bhattacharya $S$ (2012): Clomiphene citrate in combination with gonadotropins for controlled ovarian stimulation in women un- dergoing in vitro fertilization. Cochrane Database Syst Rev., 11: CD008528.

6) JosanoViC VP, KortDh, guarnaCCia MM, Sauer MV andLoBo RA (2011): Does the addition of clomiphene citrate or letrazole to gonadotropin treatment enhance the oocyte yield in poor responders undergoing IVF? J Assist ReprodGenet., 28: 1067-1072.

7) Schimberni M, Ciardo F, Schimberni M, Giallonardo A, De Pratti $V$ andSbracia $M$ (2016): Short gonadotropin-releasing hormone agonist versus flexible antagonist versus clomiphene citrate regimens in poor responders undergoing in vitro fertilization: a randomized controlled trial. Eur Rev Med Pharmacol Sci., 20(20):4354-61.

8) Schimbernl M, Ciardo F, Giallonardo A, Depratti V andSpracia $M$ (2016): Short Gonadotropin RH agonist versus flexible antagonist versus clomiphene citrate regimens in poor responders undergoing IVF, arandomized controlled trials.European review for medical and pharmacological sciences, 20:4354-4361.

9) Depalo $\mathrm{R}$, Jayakrishan $\mathrm{K}$, Garruti $\mathrm{G}$ et al.(2012): $\mathrm{GnRH}$ agonist versus $\mathrm{GnRH}$ antagonist in in vitro fertilization and embryo transfer (IVF/ET). ReprodBiolEndocrinol.,10:26.

10) D'Amato G, Caroppo E, Pasquadibisceglie A, Carone D, VittiA andVizzielloGM (2004): A novel protocol of ovulation induction with delayed gonadotropinreleasing hormone antagonist administration combined with high-dose recombinant follicle-stimulating hormone and clomiphene citrate for poor responders and women over 35 years. FertilSteril., 81: 1572-7.

11) Goswami SK, Das T, Chattopadhyay R, Sawhney V, Kumar J, Chaudhury K, Chakravarty BN andKabir SN (2004): A randomized single-blind controlled trial of letrozole as a low-cost IVF protocol in women with POR: a preliminary report, Hum Reprod., 19: 2031-2035.

12) Homburg $R$ (2005): Clomiphene citrate -end of era? Amini-review.Hum Reprod.,20:2043-51.

13) Sereepapong W, Suwajanakorn S, Triratanachat $\mathrm{S}$ et al. (2000):effect of clomiphene citrate on the endometrium of regularly cycling women.fertilysteril.,73:287-91.

14) Chaube SK, Prasad PV, Thakur SC et al. (2005): Estradiol protect clomiphene citrate induced apoptosis in ovarian follicular cells and ovulated cumulus-oocyte complexes. Fertile steril., 84:116372.

15) ozCan-CenksoY P, FiCiCiogLu C, kiziLkaLeO, suh-ha BostanCiM, BakaCakM, YesiLaDaLiM, kasPar C (2014): The comparison of the effect of microdoseGnRH-a falre up, GnRH antagonist7aromatase inhibitor letrozole and GnRH antagonist/clomi- phene citrate protocols on IVF outcomes in poor responder patients. GynecolEndocrinol., 30: 485-489. 